\title{
miR-484: A Possible Indicator of Drug-Induced Pulmonary Fibrosis
}

Takashi Konaka, Masashi Kawami, Ayano Yamamoto, Ryoko Yumoto, Mikihisa Takano

Department of Pharmaceutics and Therapeutics, Graduate School of Biomedical and Health Sciences, Hiroshima University, Hiroshima, Japan

Corresponding author: Mikihisa Takano, Ph.D., Department of Pharmaceutics and Therapeutics, Graduate School of Biomedical and Health Sciences, Hiroshima University; 1-2-3 Kasumi, Minami-ku, Hiroshima, Japan 734-8553; TEL: (+81) 82-257-5315; Fax: (+81) 82257-5319) e-mail:takanom@hiroshima-u.ac.jp

Received, September 14, 2020; Revised, November 23, 2020; Accepted, November 14, 2020; Published, November 28, 2020

\begin{abstract}
Background: Drug-induced lung injury leads to serious lung diseases, such as pulmonary fibrosis. We demonstrated in an alveolar epithelial cell line A549/ABCA3 that certain microRNAs were associated with bleomycin induced epithelial-mesenchymal transition (EMT) which is closely related to pulmonary fibrosis. In this study, we focused on the role of miR-484 in drug-induced EMT using A549/ABCA3 cells and a mouse lung injury model. Methods: The expression of EMT-related genes and miR-484 was detected by real-time polymerase chain reaction. miR-484-targeted proteins were analyzed by Western blot. Pulmonary fibrosis mouse model was prepared by the intratracheal administration of BLM. As miR-484 is known to target SMAD2 and zinc finger Ebox binding homeobox 1 (ZEB1), which are the well-known EMT-related transcription factors, we assessed the effects of a miR-484 inhibitor or mimic on the mRNA/protein expression of both the factors. Results: We found that bleomycin significantly suppressed the intracellular expression and extracellular release of miR-484 in A549/ABCA3 cells. Moreover, the miR-484 mimic and inhibitor showed no drastic effects on the expression of the EMT-related transcription factors. In addition, the miR-484 mimic had no effect on the bleomycin-induced altered mRNA expression of the $\alpha$-smooth muscle actin, a representative EMT marker. This suggested that miR484 did not directly contribute to bleomycin-induced EMT in A549/ABCA3 cells. In contrast, the significant decrease in miR-484 expression in the lung tissue or plasma of bleomycin-administered mice suggested that miR484 expression was closely correlated with bleomycin-induced lung injury. Conclusions: These findings indicate that miR-484 could be a novel diagnostic indicator for drug-induced pulmonary fibrosis.
\end{abstract}

\section{INTRODUCTION}

MicroRNAs (miRNAs) are noncoding small RNAs that are approximately $18-25$ nucleotides in length. Mature miRNAs have been recognized as important regulators of the corresponding gene expressions [1]. In general, miRNAs bind to the $3^{\prime}$-untranslated regions (UTRs) of mRNAs and regulate subsequent protein synthesis. In addition, miRNAs interact with the 5'-UTR and the coding sequence of target mRNAs and thus lead to cleavage and degradation of the mRNAs. Thus, miRNAs are possibly associated with the regulation of gene expression and protein synthesis related to various physiological and/or pathophysiological reactions in the body. For instance, it has been reported that the miRNAs in exosomes secreted by various cells, including cancer cells, are involved in cancer progression and metastasis [2,3]. Therefore, clinical applications of miRNAs as therapeutic agents or biomarkers in cancer treatment are currently under investigation.

Pulmonary fibrosis (PF) is a chronic respiratory disease, in which the lung tissue is scarred, thickened, and stiff, resulting in reduced oxygen supply to the blood. Anticancer drugs, including bleomycin, induce PF more readily than other agents such as antimicrobial drugs, antirheumatic drugs, and interferons [4]. PF is commonly associated with abnormal tissue repair in damaged alveolar epithelial type II cells [5]; an excessive extracellular matrix (ECM), including collagen, plays a crucial role in the pathogenesis of PF [6]. In this condition, myofibroblasts producing ECM are recruited from the damaged epithelial cells through the epithelialmesenchymal transition (EMT) [7]. Therefore, mechanistic investigation regarding the association of EMT with PF may lead to the development of a novel therapeutic approach against severe lung diseases. To date, however, the relationship between PF and EMT has not been fully elucidated. In particular, the concept of drug-induced EMT is not well understood.

Recent studies have demonstrated alterations in the expression of several miRNAs in patients with PF $[8,9]$. Moreover, increasing evidence suggests that 
several miRNAs play an important role in EMT [10, 11 ], suggesting that miRNAs could be the key factors to clarify the mechanism underlying drug-induced EMT. Notably, the miR-200 family is well known to suppress EMT via the regulation of EMT-related transcription factors, including zinc finger E-boxbinding homeobox (ZEB) 1 and 2 [12]. We have also demonstrated using several alveolar epithelial cell lines that miR-34a directly contributes to EMT induction [13, 14]. Thus, miRNAs should be highlighted as one of the crucial factors associated with drug-induced EMT.

Recently, it has been reported that miR-484 inhibits EMT by targeting EMT-related genes such as SMAD2 and ZEB1 in cervical cancer cells. In addition, the long noncoding RNA H19 has been reported to promote EMT in A549 cells by suppressing miR-484 expression $[15,16]$, thus suggesting a significant contribution of miR-484 to the EMT process. To date, however, the involvement of miR-484 in drug-induced EMT has not been examined. A549/ABCA3 cells are established by introducing a retrovirus vector that includes ABCA3 gene into A549, a human-derived cultured alveolar epithelial cell line, and we have confirmed that upregulation of type II alveolar epithelium phenotypes such as increase in formation of lamellar bodies and peptide transporter 2 expression in the cells [14]. In addition, BLM significantly induces EMT in the cells [14], indicating that A549/ABCA3 cells would be useful for evaluate drug-induced EMT in the lung. In the present study, we examined, for the first time, the role of miR-484 in bleomycin-induced EMT using an alveolar epithelial cell line, A549/ABCA3, and using the lung tissues of a mouse lung injury model established following the intratracheal administration of bleomycin. Our study provides novel findings concerning miR-484, which can be developed as a possible diagnostic indicator for drug-induced EMT and lung injury.

\section{MATERIALS AND METHODS}

Materials. Bleomycin was purchased from Tokyo Chemical Industry Co., LTD. (Tokyo, Japan). All the other chemicals used in the experiments were obtained from commercial sources and were of the highest purity.

Cell culture. A549/ABCA3 cells was established by transfecting a plasmid DNA including the $A B C A 3$ gene into A549 cells, a lung alveolar epithelial cell line, using a retroviral vector; the cells were then cultured as reported previously [14].

Drug treatment and transfection with the miR484 mimic or inhibitor. A549/ABCA3 cells were cultivated on 12-well plates at a cell density of $1 \times 10^{5}$ cells/well for real-time polymerase chain reaction (PCR) or in a $60-\mathrm{mm}$ dish at a cell density of $5 \times 10^{5}$ cells/dish for Western blot analysis. These cells were treated with $60-\mu \mathrm{M}$ bleomycin for $72 \mathrm{~h}$ or $144 \mathrm{~h}$. During cell seeding, $10 \mathrm{nM}$ of a miR-484 mimic HsamiR-484 (QIAGEN, Hilden, Germany) or $20 \mathrm{nM}$ of an miR-484 inhibitor Anti-hsa-miR-484 (QIAGEN) were transfected using Lipofectamine2000 (Thermo Fisher Scientific Inc., MA) for $24 \mathrm{~h}$.

In the case of combinatorial drug treatment with a miR-484 mimic, the miR-484 mimic was first transfected for $24 \mathrm{~h}$. After that, the cells were treated with $60-\mu \mathrm{M}$ BLM for $48 \mathrm{~h}$.

Real-time PCR for miRNA detection. Detection of miRNAs was performed as reported previously [13, 14]. Briefly, miRNA was extracted from A549/ABCA3 cells and lung tissues of mice using the High Pure miRNA Isolation Kit (Roche Diagnostics, Laval, QC, Canada) or from the supernatants of treated cells and plasma from mice using the miRNeasy Serum/Plasma Kit (QIAGEN). miRNA reverse transcription with complementary DNA and its quantitative real-time PCR were performed as reported previously $[13,14]$. The primers for miR-484-5p, RNU6-2, and miR-39 derived from Caenorhabditis elegans (spike-in control) were purchased from QIAGEN. The expression of miRNA was normalized to that of RNU6-2 or miR-39.

Real-time PCR for mRNA detection. mRNA expression was assessed as reported previously [17]. The extraction of total RNA from the treated cells, reverse transcription, and amplification of the obtained cDNA were performed as reported previously [17]. The primers used were as follows: sense, 5'-AGCAGGAATTGAGCCACAGA-3' and antisense, 5'-CAGGGGAAAGAGTAGTAGGAGA3' for SMAD2; sense, 5'AGCTGCCAATAAGCAAACGA-3' and antisense, 5'-TTTGGGCGGTGTAGAATCAG-3' for ZEB1. The primers for $\alpha$-SMA and glyceraldehyde-3phosphate dehydrogenase (GAPDH) were prepared as reported previously [14]. The expression of mRNA was normalized to that of GAPDH, a housekeeping gene. 
Western blot analysis. Protein expression was examined by Western blot analysis. Briefly, proteins were extracted from A549/ABCA3 cells using radioimmunoprecipitation assay buffer $(150-\mathrm{mM}$ $\mathrm{NaCl}$, 5-mM EDTA, 1-mM phenylmethanesulfonyl fluoride, 50-mM Tris, $1 \%$ NP-40, $0.1 \%$ sodium dodecyl sulfate [SDS], $1 \%$ sodium deoxycholate; $\mathrm{pH}$ 7.4). The protein extracts for SMAD2 or ZEB1 analysis were first subjected to SDS-polyacrylamide gel electrophoresis using $10 \%$ polyacrylamide gels and then transferred to the polyvinylidene difluoride membrane. After blotting, the membrane was blocked with $5 \%$ skim milk and subsequently incubated for $2 \mathrm{~h}$ with primary antibodies against SMAD2 (1:300 dilution) (Santa Cruz Biotechnology, Inc., Dallas, TX, USA), ZEB1 (1:1000) (BIO-RAD, Tokyo, Japan), and GAPDH (1:5000) (SigmaAldrich, St. Louis, MO, USA). The membrane was then washed three times using Tris-buffered saline with Tween (TBS-T) (20.5-mM Tris, $150-\mathrm{mM} \mathrm{NaCl}$, $0.05 \%$ Tween 20; pH 7.5) and then incubated for $1 \mathrm{~h}$ with horseradish peroxidase (HRP)-labeled secondary antibodies (1:5000 dilution for GAPDH and ZEB1, 1:2500 dilution for SMAD2). After washing three times with TBS-T, the antibody complexes were visualized using Luminata Forte Western HRP substrate reagents (Merck Millipore).

PF animal model. Male Slc ddy mice with ages of 3 weeks were purchased from Japan SLC, Inc. (Hamamatsu, Shizuoka, Japan). The study protocol was approved by the Committee of Research Facilities for Laboratory Animal Sciences, Hiroshima University, and experiments with animals were performed in accordance with the Guide for Animal Experimentation from Hiroshima University. Briefly, Slc ddy mice (control; $n=3$, bleomycin; $n=3$ 4) were housed under standard conditions with free access to water and rodent food. Saline or bleomycin $(5 \mathrm{mg} / \mathrm{kg})$ were intratracheally administered into the mice at day 0 and sacrificed at day 14 or 21 for obtaining the lung tissue homogenates.

Hydroxyproline assay. Collagen accumulation in the lungs was evaluated by measuring the amount of hydroxyproline using the hydroxyproline assay kit (Perchlorate-free) (CELL BIOLABS, INC.). Lung tissue homogenates were hydrolyzed in $12 \mathrm{~N}$ hydrochloric acid at $120^{\circ} \mathrm{C}$ for $3 \mathrm{~h}$. The solution was removed by blowing nitrogen gas, after which the Chloramine T Mixture (Chloramine T Reagent and Assay buffer) was added. The mixtures were incubated at room temperature $\left(20-25^{\circ} \mathrm{C}\right)$ for $30 \mathrm{~min}$ and, finally, Ehrlich's Reagent was added to each solution. The solutions were then heated at $60{ }^{\circ} \mathrm{C}$ for $45 \mathrm{~min}$, and the absorbance was read at $556 \mathrm{~nm}$ using a microplate reader. Hydroxyproline standard curve was plotted using serial dilutions in the concentration range of $0-100 \mu \mathrm{g} / \mathrm{mL}$.

Statistical analysis. Data are expressed as means \pm standard errors of the mean. Statistical analysis was performed using one-way analysis of variance followed by Tukey's test for multiple comparisons or by the Student's $t$-test. The level of significance was set at $* \mathrm{p}<0.05$ or $* * \mathrm{p}<0.01$.

\section{RESULTS}

Effect of bleomycin on miR-484 expression in A549/ABCA3 cells and supernatants. The effect of BLM on the intracellular and extracellular expression of miR-484 was examined. In a previous report, we demonstrated that bleomycin induced EMT in A549/ABCA3 cells [14]. In this study, we observed significant decreases in the intracellular expression and extracellular release of miR-484 in bleomycin-treated cells (Figure 1), suggesting that bleomycin treatment may suppress the synthesis of miR-484, followed by the reduction of miR-484 release in the extracellular space.

\section{Contribution of miR-484 to EMT-related factors} in A549/ABCA3 cells. To investigate the contribution of miR-484 to the EMT process, the effect of an miR-484 inhibitor on EMT-related factors was examined. The introduction of an miR484 inhibitor into the A549/ABCA3 cells markedly decreased the miR-484 expression in the cells but showed no effect on the mRNA expression of $\alpha$ SMA (Figure 2A, B). As it has been reported that miR-484 regulates EMT via the control of SMAD2 and ZEB1 (EMT-related transcription factors) expression [16], we further investigated the effect of the miR-484 inhibitor on the mRNA/protein expression of these transcription factors. However, SMAD2 and ZEB1 expression was not affected by transfection with a miR-484 inhibitor (Figure 2C, D, E). Next, the effect of miR-484 mimic transfection on EMT-related factors was examined. As shown in Figure $3 \mathrm{~A}$, the introduction of a miR-484 mimic markedly upregulated miR-484 expression level in A549/ABCA3 cells. However, no significant change was noted in $\alpha$-SMA mRNA expression following transfection with the mimic (Figure 3B). Furthermore, the mimic had no effect on the 
mRNA/protein expression of SMAD2 and ZEB1 (Figure 3C, D, E). Therefore, the direct contributions of miR-484 to EMT-related factors were not observed under the conditions used in this study.
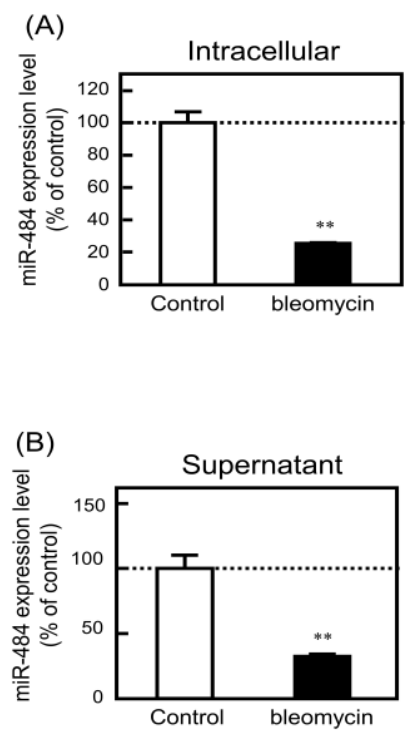

Figure 1. Effect of bleomycin $(60 \mu \mathrm{M})$ on intracellular and extracellular expression levels of miR-484 in A549/ABCA3 cells. The cells were treated with $60 \mu \mathrm{M}$ bleomycin for $144 \mathrm{~h}$ (A) or $72 \mathrm{~h}$ (B). After bleomycin treatment, the cells or the supernatant components via lyophilization were dissolved with lysis buffer for preparation of total RNA, respectively. The miR-484 expression level was measured by real-time PCR analysis as described in Materials and Methods. The dashed line indicates the control level. Each value represents the mean \pm S.E.M $(\mathrm{n}=3) . * * \mathrm{p}<0.01$ : significantly different from each control.
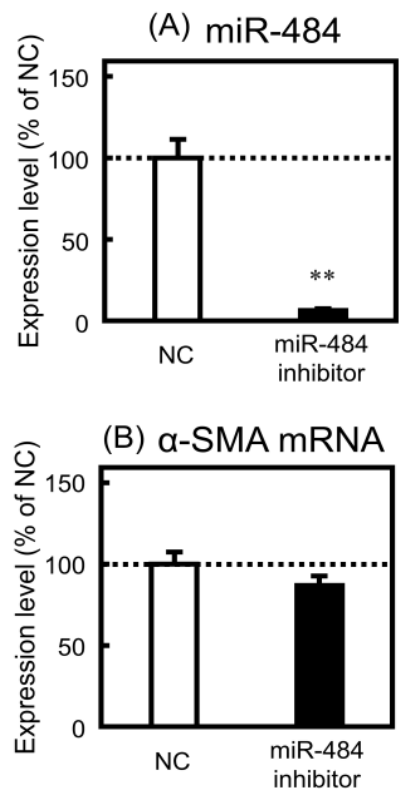

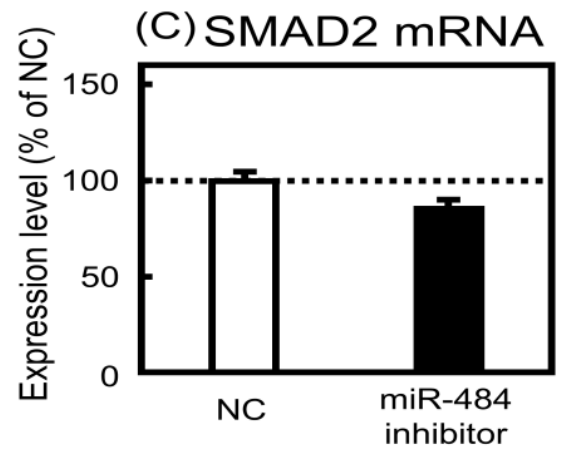

(D) ZEB1 mRNA

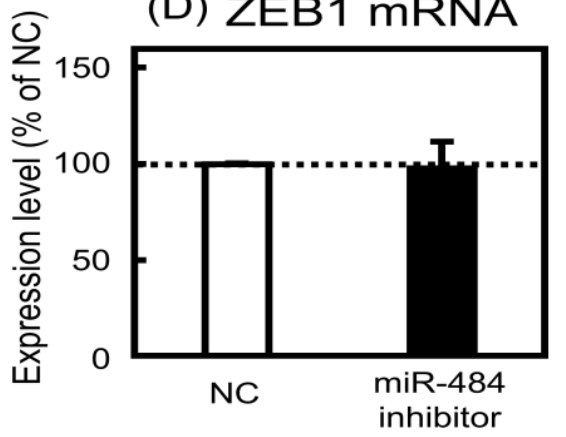

(E)

miR-484 inhibitor

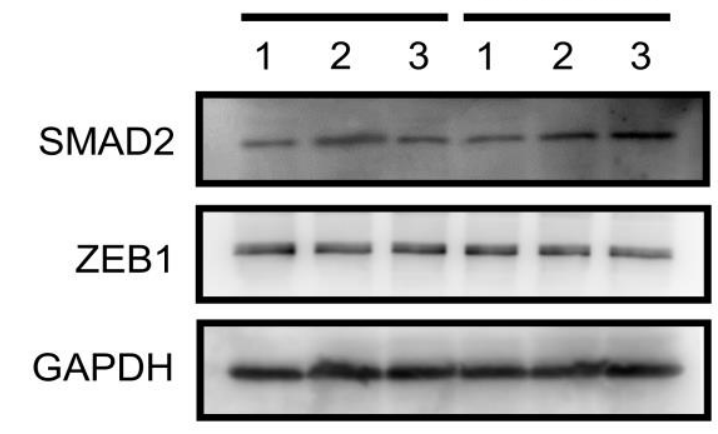

Figure 2. Effect of miR-484 inhibitor on the expressions of EMT-related factors in A549/ABCA3 cells. The cells were transfected with negative control (NC) or miR-484 inhibitor $(20 \mathrm{nM})$ for $24 \mathrm{~h}$. After $72 \mathrm{~h}$, the expression levels of miR-484 (A), and mRNAs of $\alpha$-SMA (B), SMAD2 (C), and ZEB1 (D) were measured by real-time PCR analysis. (E) The protein expressions of SMAD2 and ZEB1 were measured by Western blot analysis. The dashed line indicates the negative control (NC) level. Each value represents the mean \pm S.E.M $(n=3)$. ${ }^{* *} \mathrm{p}<0.01$, significantly different from NC.

Figure 2 continues ... 

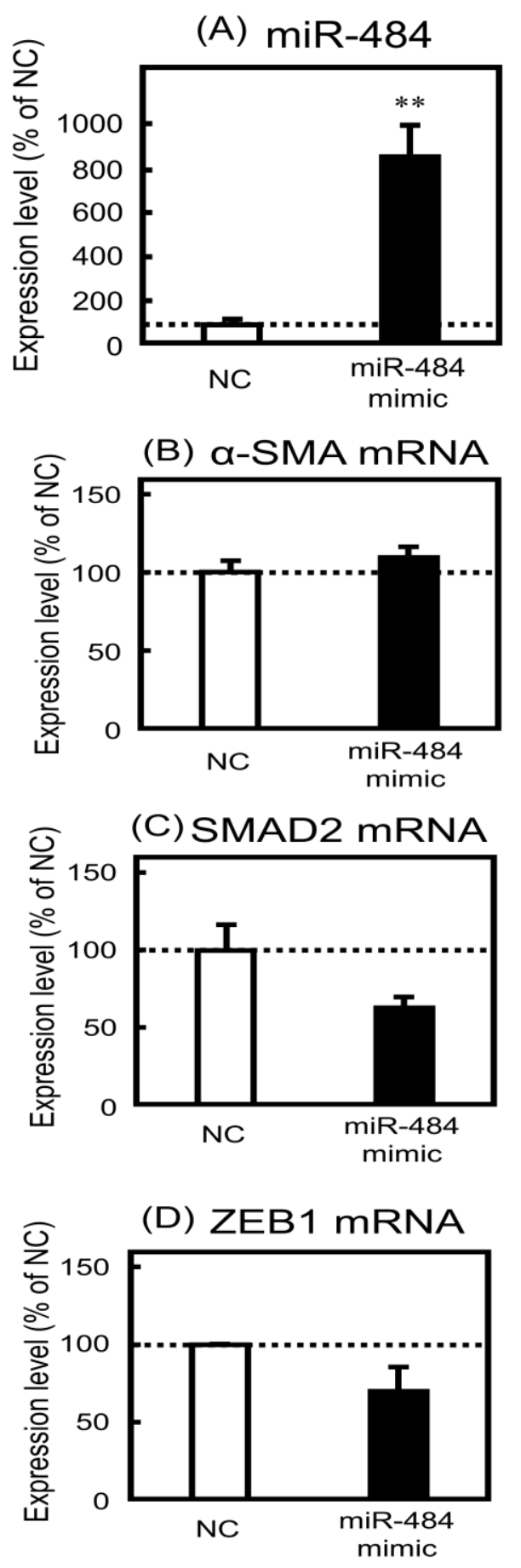

Figure 3 continues...

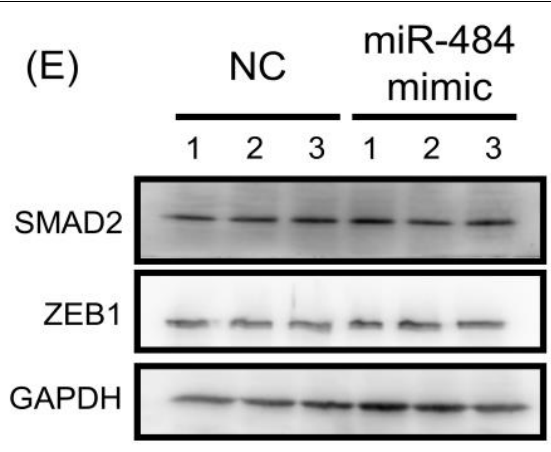

Figure 3. Effect of miR-484 mimic on the expressions of EMT-related factors in A549/ABCA3 cells. The cells were transfected with negative control (NC) or miR-484 mimic $(10 \mathrm{nM})$ for $24 \mathrm{~h}$. After $48 \mathrm{~h}$, the expression levels of miR-484 (A), and mRNAs of $\alpha$-SMA (B), SMAD2 (C), and ZEB1 (D) were measured by real-time PCR analysis. (E) The protein expressions of SMAD2 and ZEB1 were measured by Western blot analysis. The dashed line indicates the negative control (NC) level. Each value represents the mean \pm S.E.M $(n=3)$. **p $<0.01$, significantly different from NC.

Role of miR-484 in bleomycin-induced EMT in A549/ABCA3 cells. To clarify the role of miR-484 in bleomycin-induced EMT in A549/ABCA3 cells, we examined the effect of the miR-484 mimic on bleomycin-induced upregulation of $\alpha$-SMA mRNA expression. As shown in Figure 4A, bleomycin had no effect on the increase in miR-484 expression induced by mimic introduction. In addition, bleomycin-induced increase in $\alpha$-SMA mRNA expression was not affected by the introduction of the miR-484 mimic (Figure 4B). This indicated that miR-484 may not be involved in bleomycin-induced EMT in A549/ABCA3 cells.

Relationship between EMT and miR-484 in bleomycin-treated lung injury mouse model. We established a mouse model of lung injury by intratracheally administering bleomycin. On day 14 or 21 after the administration, lung tissues were obtained from the mice administered saline (control) or bleomycin. As shown in Figure 5A, the mRNA expression of colla1, which encodes collagen type IA 1 , increased on day 21 in bleomycin-treated mice compared with that in control mice. In general, the level of hydroxyproline is regarded as an indicator of the collagen accumulation, because hydroxyproline is a major component of collagen [18]. The amounts of hydroxyproline in the lung tissue was also upregulated on day 21 (Figure 5B), indicating that 
the bleomycin-induced lung injury mouse model was successfully established. In contrast, the mRNA expression of $\alpha$-SMA increased on day 14, but not on day 21 (Figure 5C), which was not comparable to the trend observed for colla1 and hydroxyproline. On day 21 , the expression of miR-484 was examined in lung tissues and plasma. Significant decreases in the expression of miR-484 in lung tissues and plasma were observed (Figure 5D), indicating that miR-484 might be related to bleomycin-induced lung injury.
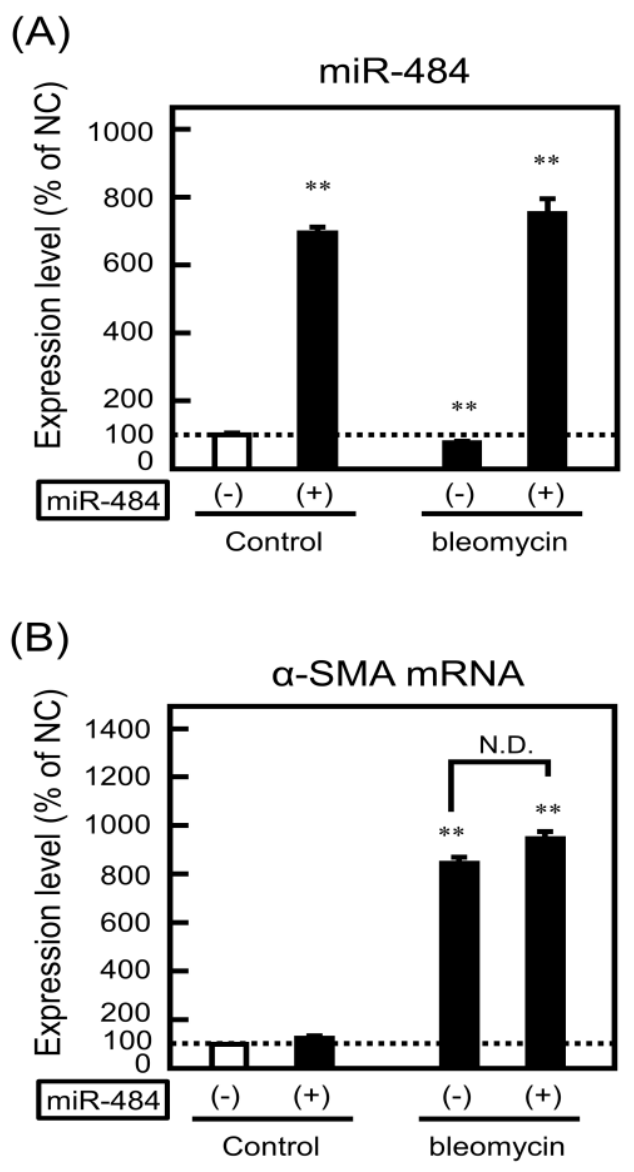

Figure 4. Role of miR-484 in bleomycin-induced EMT in A549/ABCA3 cells. The cells were transfected with negative control (-) or miR-484 mimic (+) for $24 \mathrm{~h}$. After that, the cells were treated without (Control) or with bleomycin $(60 \mu \mathrm{M})$ for $48 \mathrm{~h}$. The expression levels of miR-484 (A) and $\alpha$-SMA mRNA (B) were measured by real-time PCR analysis. The dashed line indicates the negative control (NC) in Control. Each value represents the mean \pm S.E.M $(n=3)$. $* * p<0.01$, vs NC in control.

\section{DISCUSSION}

EMT is currently recognized as an important step that promotes the pathogenesis of severe lung diseases including PF. However, more research is required to fully understand the relationship between EMT and lung diseases. In particular, drug-induced EMT seems to have a complex mechanism owing to the interaction of the drug with various factors other than the EMT process. At present, there is limited information regarding the clinical therapy, preventive methods, and effective diagnosis for pulmonary disorders caused by drugs. Therefore, more evidence is required for the development of therapeutic agents, predictable biomarkers, and robust diagnosis indicators against drug-induced lung injuries. In contrast, miRNAs have been highlighted as therapeutic targets and effective biomarkers for various diseases [19, 20]. In addition, we have already demonstrated that miR-34a directly contributes to EMT promotion in A549/ABCA3 cells [14], suggesting that miRNAs are the key factors for clarifying the mechanism underlying drug-induced lung injury and a possible clinical tool. Here, we focused on the role of miR-484 in bleomycininduced EMT using an alveolar epithelial cell line, A549/ABCA3, and a bleomycin-induced mouse lung injury model. To the best of our knowledge, our study is the first to provide evidence concerning the relationship between miR-484 and drug-induced EMT in the lungs.
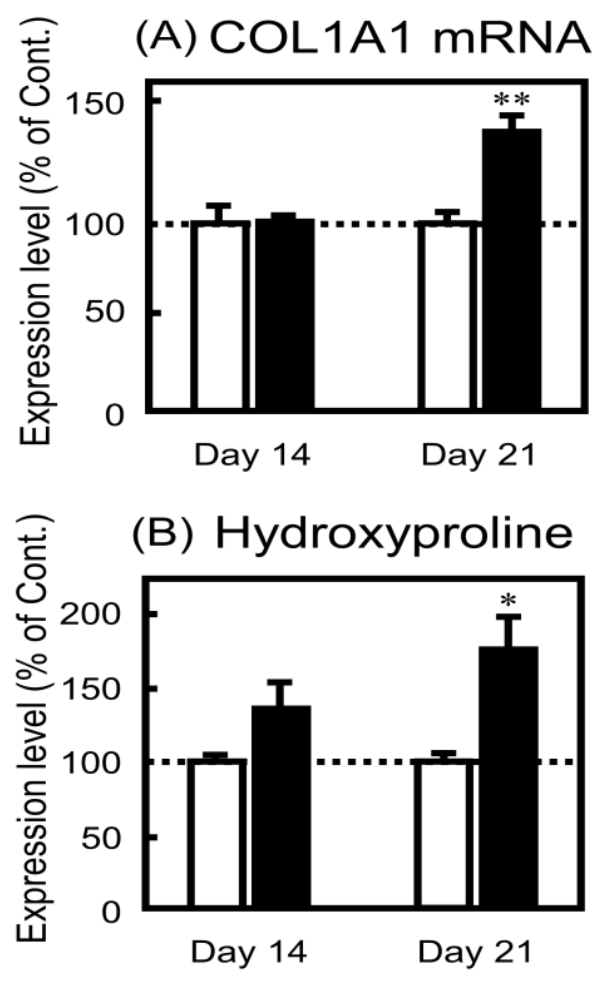

Figure 5 continues... 

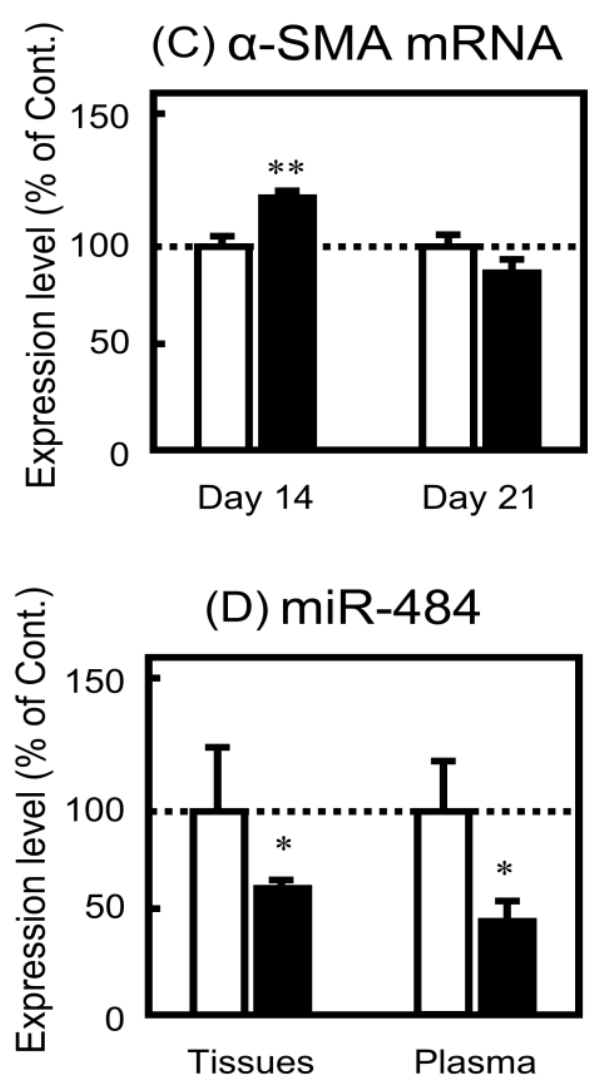

Figure 5. Effect of intratracheal administration of bleomycin on the expression levels of COL1A1 mRNA (A), hydroxyproline (B), $\alpha$-SMA mRNA (C), and miR484 (D) in the mice. $5 \mathrm{mg} / \mathrm{kg}$ bleomycin were administered into male Slc ddy mice at day 0, and sacrificed at day 14 or 21. The expression of miR-484 and mRNA expression levels of COL1A1 and $\alpha$-SMA were analyzed by real-time PCR. The amounts of hydroxyproline were evaluated by the assay kit as described in Materials and Methods. The dashed line indicates the control level. Each value represents the mean \pm S.E.M $(\mathrm{n}=3-4)$. $* \mathrm{p}<0.05$ : significantly different from the control.

Although several research groups, including our own, have elucidated bleomycin-induced EMT, different concentrations of bleomycin with a large range $(0.4-120 \mu \mathrm{M})$ were used to induce EMT in those studies [14, 21-26]. For example, we used 60$\mu \mathrm{M}$ bleomycin to induce EMT in A549 and A549/ABCA3 cells [14, 24], while $0.4 \mu \mathrm{M}$ bleomycin induced EMT-like phenotypical changes of RLE/Abca3 cells derived from rat normal alveolar epithelium [21]. In preliminary experiments, we confirmed that low concentrations $(0.12-4 \mu \mathrm{M})$ of bleomycin had no effects on mRNA expression levels of EMT-related genes in A549 cells (data not shown). Therefore, EMT-inducing effect of bleomycin may depend on the property of the cell types. On the other hand, one clinical study reported that serum concentration of bleomycin after single bolus injection of bleomycin was $2.213 \pm 0.0911 \mu \mathrm{M}$ [27], which is quite different from the present experimental condition, whereas the higher concentration of bleomycin should be required to detect the EMT-like phenotypical changes under certain in vitro conditions. Thus, there remain limitations to fill the gap between the clinical situation and the experimental conditions regarding the bleomycin-induced EMT.

miR-484 has multifunctional characteristics and contributes to many physical and pathologic processes. For example, it has been reported that miR-484 enhances the sensitivity of several chemotherapeutic agents by targeting cytidine deaminase and vascular endothelial growth factor in breast cancer cells and ovarian carcinomas, respectively [28,29]. In addition, it has been demonstrated that miR-484 inhibits the EMT process via the suppression of ZEB1 and SMAD2 expression in ovarian cancer cell lines [16]. These findings prompted us to examine the role of miR-484 in bleomycin-induced EMT in the alveolar epithelial cell line A549/ABCA3. As expected, bleomycin treatment significantly decreased the intracellular expression and extracellular release of miR-484 in the cells. However, treatment with the miR-484 inhibitor and mimic had no effect on the mRNA expression of $\alpha$-SMA, as well as on the mRNA/protein expression of ZEB1 and SMAD2. Furthermore, bleomycin-induced EMT was not affected by miR-484 mimic introduction, suggesting that miR-484 is not involved in the EMT process triggered by bleomycin in A549/ABCA3 cells, which is different from the observations in ovarian cancer cells. These differences may be attributed to the different types of cell lines, warranting investigation of mechanisms contributing to these differences in future studies.

Considering that the intracellular expression and extracellular release of miR-484 are well correlated with bleomycin-induced EMT in A549/ABCA3 cells, miR-484 may serve as an effective indicator of druginduced lung injury. Accordingly, we established a bleomycin-induced lung injury mouse model and examined the expression of miR-484 in mouse lung tissue and plasma. Interestingly, the mRNA expression of $\alpha$-SMA, an EMT indicator, was significantly higher in bleomycin-administered mice than that in the control mice on day 14 but not on day 21. Conversely, the mRNA expression of colla1 and 
the level of hydroxyproline (fibrosis markers) increased in bleomycin-administered mice on day 21 , but not on day 14, compared with those in control mice. These trends were comparable with those observed in previous reports [30,31], suggesting the gap in the dynamics of EMT and the development of fibrosis. On day 21 after the intratracheal administration of bleomycin, $\mathrm{miR}-484$ expression in lung tissues and plasma significantly decreased. Therefore, miR-484 could be developed as a novel diagnostic indicator for drug-induced lung injury. Furthermore, miR-34a expression was upregulated in the plasma of bleomycin-administered mice (data not shown), and this result was similar to that observed in our previous in vitro study $[13,14]$.

Recent studies have demonstrated that serum miR-484 is increased in patients with non-small cell lung carcinoma (NSCLC) [32] and that miR-484 promotes the progression of NSCLC [33], indicating that this miRNA may be involved in cancer progression. Thus, miR-484 may be involved in various pathological conditions other than EMTrelated disorders. Therefore, the use of only miR-484 as an indicator of drug-induced lung injury should be avoided. Moreover, we found that miR-34a was also increased in the plasma of bleomycin-treated model mice (data not shown). Thus, the use of multiple diagnostic markers, including several miRNAs related to the EMT process, may be an effective approach to determine the pathological status of lung injuries. Further studies are needed to establish effective and robust diagnostic methods using miRNAs for drug-induced lung injury.

\section{CONCLUSION}

Our study is the first to determine that bleomycin treatment induced a decrease in the intracellular expression and extracellular release of miR-484 in A549/ABCA3 cells. In contrast, the introduction of a miR-484 inhibitor or mimic into the cells had no effect on the mRNA/protein expression of EMTrelated factors such as zinc finger E-box binding homeobox 1 and SMAD2. In addition, bleomycininduced increase in the mRNA expression of $\alpha$ smooth muscle actin was not affected by the miR-484 mimic. This suggested that miR-484 expression is associated with bleomycin-induced epithelialmesenchymal transition (EMT) and not with the EMT process triggered by bleomycin in cells; this is a unique and novel finding concerning the relationship between miR-484 and bleomycininduced EMT. Further in vivo study revealed that
miR-484 expression significantly decreased in the lung tissues and plasma of bleomycin-induced mice with lung injury. These findings suggest that miR484 is a novel diagnostic indicator of drug-induced EMT, a precursor to serious lung diseases such as pulmonary fibrosis.

\section{ACKNOWLEDGEMENT}

This study was supported in part by the Grants-inAid for Scientific Research from the Japan Society for the Promotion of Science (JP18H02586, JP18K06749, and JP19K16447).

\section{CONFLICT OF INTEREST}

The authors declare that there are no conflicts of interest.

\section{REFERENCES}

1. Bartel DP, MicroRNAs: Target Recognition and Regulatory Functions. Cell, 2009;136:215-233. doi:10.1016/j.cell.2009.01.002

2. McDonald MK, Capasso KE, Ajit SK, Purification and microRNA profiling of exosomes derived from blood and culture media. J Vis Exp, 2013;76:e50294 doi:10.3791/50294

3. Vanni I, Alama A, Grossi F, Dal Bello MG, Coco S, Exosomes: a new horizon in lung cancer. Drug Discov Today, 2017;22:927-936. doi:10.1016/j.drudis.2017.03.004

4. Kubo K, Azuma A, Kanazawa M, Kameda H, Kusumoto M, Genma A, Saijo Y, Sakai F, Sugiyama Y, Tatsumi K, Dohi M, Tokuda H, Hashimoto S, Hattori N, Hanaoka M, Fukuda Y, Consensus statement for the diagnosis and treatment of druginduced lung injuries. Respir Investig, 2013;51:260277. doi:10.1016/j.resinv.2013.09.001.

5. Iwano M, Plieth D, Danoff TM, Xue C, Okada H, Neilson EG, Evidence that fibroblasts derive from epithelium during tissue fibrosis. J Clin Invest, 2002;110:341-350. doi:10.1172/JCI15518.

6. Upagupta C, Shimbori C, Alsilmi R, Kolb M, Matrix abnormalities in pulmonary fibrosis. Eur Respir Rev, 2018;27:180033. doi: 10.1183/16000617.0033-2018.

7. Gharaee-Kermani M, Hu B, Phan SH, Gyetko MR, Recent advances in molecular targets and treatment of idiopathic pulmonary fibrosis: focus on TGFbeta signaling and the myofibroblast. Curr Med Chem, 2009;16:1400-1417. doi:10.2174/092986709787846497.

8. Rajasekaran S, Rajaguru P, Sudhakar Gandhi PS, MicroRNAs as potential targets for progressive pulmonary fibrosis. Front Pharmacol, 2015;6:254. doi:10.3389/fphar.2015.00254. 
9. Li H, Zhao X, Shan H, Liang H, MicroRNAs in idiopathic pulmonary fibrosis: involvement in pathogenesis and potential use in diagnosis and therapeutics. Acta Pharm Sin B, 2016;6:531539. doi:10.1016/j.apsb.2016.06.010.

10. Musavi Shenas MH, Eghbal-Fard S, Mehrisofiani V, Abd Yazdani N, Rahbar Farzam O, Marofi F, Yousefi M, MicroRNAs and signaling networks involved in epithelial-mesenchymal transition. J Cell Physiol, 2019;234:5775-5785. doi:10.1002/jcp.27489.

11. Zou XZ, Liu T, Gong ZC, Hu CP, Zhang Z, MicroRNAs-mediated epithelial-mesenchymal transition in fibrotic diseases. Eur J Pharmacol, 2017;796:190-206.

doi:10.1016/j.ejphar.2016.12.003.

12. Park SM, Gaur AB, Lengyel E, Peter ME, The miR200 family determines the epithelial phenotype of cancer cells by targeting the E-cadherin repressors ZEB1 and ZEB2. Genes Dev, 2008;22:894-907. doi:10.1101/gad.1640608.

13. Takano M, Nekomoto C, Kawami M, Yumoto R, Role of miR-34a in TGF- $\beta 1$ - and drug-induced epithelial-mesenchymal transition in alveolar type II epithelial cells. J Pharm Sci, 2017;106:2868-2872. doi:10.1016/j.xphs.2017.04.002.

14. Yamamoto A, Kawami M, Konaka T, Takenaka S, Yumoto R, Takano M, Anticancer drug-induced epithelial-mesenchymal transition via p53/miR-34a axis in A549/ABCA3 cells. J Pharm Pharm Sci, 2019;22:516-524. doi:10.18433/jpps30660.

15. Zhang Q, Li X, Li X, Li X, Chen Z, LncRNA H19 promotes epithelial-mesenchymal transition (EMT) by targeting miR-484 in human lung cancer cells. J Cell Biochem, 2018;119:4447-4457. doi:10.1002/jcb.26537.

16. Hu Y, Xie H, Liu Y, Liu W, Liu M, Tang H, miR484 suppresses proliferation and epithelialmesenchymal transition by targeting ZEB1 and SMAD2 in cervical cancer cells. Cancer Cell Int, 2017;17:36. doi:10.1186/s12935-017-0407-9.

17. Kawami M, Harabayashi R, Miyamoto M, Harada R, Yumoto R, Takano M, Methotrexate-induced epithelial-mesenchymal transition in the alveolar epithelial cell line A549. Lung, 2016;194:923-930. doi:10.1007/s00408-016-9935-7.

18. Kliment CR, Englert JM, Crum LP, Oury TD, A novel method for accurate collagen and biochemical assessment of pulmonary tissue utilizing one animal. Int J Clin Exp Pathol, 2011;4:349-355.

19. Dwivedi S, Purohit P, Sharma P, MicroRNAs and Diseases: Promising Biomarkers for Diagnosis and Therapeutics. Indian J Clin Biochem, 2019;34:243245. doi:10.1007/s12291-019-00844-x.

20. Walayat A, Yang M, Xiao D, Therapeutic Implication of miRNA in Human Disease: Antisense Therapy. IntechOpen, 2019. doi:10.5772/intechopen.82738.
21. Takano M, Yamamoto C, Yamaguchi K, Kawami M, Yumoto R, Analysis of TGF- $\beta 1$ - and drug-induced epithelial-mesenchymal transition in cultured alveolar epithelial cell line RLE/Abca3. Drug Metab Pharmacokinet, 2015;30:111-118. doi:10.1016/j.dmpk.2014.10.007.

22. Chen LJ, Ye H, Zang Q, Li FZ, Song LJ, Mu Q, Rao SS, Cai PC, Xiang F, Zang JC, Su Y, Xin JB, Ma WL, Bleomycin induced epithelial-mesenchymal transition (EMT) in pleural mesothelial cells. Toxicol Appl Pharmacol, 2015;283:75-82. doi:10.1016/j.taap.2015.01.004.

23. Tian R, Zhu Y, Yao J, Meng X, Wang J, Xie H, Wang $\mathrm{R}$, NLRP3 participates in the regulation of EMT in bleomycin-induced pulmonary fibrosis. Exp Cell Res, 2017;357:328-334. doi:10.1016/j.yexcr.2017.05.028.

24. Kawami M, Harada R, Ojima T, Yamaguchi Y, Yumoto R, Takano M, Association of cell cycle arrest with anticancer drug-induced epithelialmesenchymal transition in alveolar epithelial cells. Toxicology, 2019;424:152231. doi:10.1016/j.tox.2019.06.002.

25. Huang JJ, Xia J, Huang LL, Li YC, HIF-1 $\alpha$ promotes NJRP3 inflammasome activation in bleomycininduced acute lung injury. Mol Med Rep, 2019;20:3424-3432. doi:10.3892/mmr.2019.10575.

26. Petukhov D, Richter-Dayan M, Fridlender Z, Breuer $\mathrm{R}$, Wallach-Dayan SB, Increased regeneration following stress-induced lung injury in bleomycintreated chimeric mice with CD44 knockout mesenchymal cells.

Cells, 2019;8:1211. doi:10.3390/cells8101211.

27. Groselj A, Krzan M, Kosjek T, Bosnjak M, Sersa G, Cemazar M, Bleomycin pharmacokinetics of bolus bleomycin dose in elderly cancer patients treated with electrochemotherapy. Cancer Chemother Pharmacol, 2016;77:939-947. doi:10.1007/s00280016-3004-z.

28. Vecchione A, Belletti B, Lovat F, Volinia S, Chiappetta G, Giglio S, Sonego M, Cirombella R, Onesti EC, Pellegrini P, Califano D, Pignata S, Losito S, Canzonieri V, Sorio R, Alder H, Wernicke D, Stoppacciaro A, Baldassarre G, Croce CM, A microRNA signature defines chemoresistance in ovarian cancer through modulation of angiogenesis. Proc Natl Acad Sci U S A, 2013;110:9845-9850. doi:10.1073/pnas.1305472110.

29. Ye FG, Song CG, Cao ZG, Xia C, Chen DN, Chen L, Li S, Qiao F, Ling H, Yao L, Hu X, Shao ZM, Cytidine deaminase axis modulated by miR-484 differentially regulates cell proliferation and chemoresistance in breast cancer. Cancer Res, 2015;75:1504-1515. doi:10.1158/0008-5472.CAN14-2341.

30. Tsukui T, Ueha S, Abe J, Hashimoto S, Shichino S, Shimaoka T, Shand FH, Arakawa Y, Oshima K, Hattori M, Inagaki Y, Tomura M, Matsushima K, Qualitative rather than quantitative changes are 
hallmarks of fibroblasts in bleomycin-induced pulmonary fibrosis. Am J Pathol, 2013;183:758-773. doi:10.1016/j.ajpath.2013.06.005.

31. Rock JR, Barkauskas CE, Cronce MJ, Xue Y, Harris JR, Liang J, Noble PW, Hogan BL, Multiple stromal populations contribute to pulmonary fibrosis without evidence for epithelial to mesenchymal transition. Proc Natl Acad Sci U S A, 2011;108:E1475-83. doi:10.1073/pnas.1117988108.

32. Zhuang Z, Sun C, Gong H, High serum miR-484 expression is associated with the diagnosis and prognosis of patients with non-small cell lung cancer. Exp Ther Med, 2019;18:4095-4102. doi:10.3892/etm.2019.8010.

33. Li T, Ding ZL, Zheng YL, Wang W, MiR-484 promotes non-small-cell lung cancer (NSCLC) progression through inhibiting Apaf-1 associated with the suppression of apoptosis. Biomed Pharmacother, 2017;96:153-164. doi: 10.1016/j.biopha.2017.09.102. 